\title{
COMUNICANTES FALTOSOS DOS PORTADORES DE HANSENÍASE: UMA TRANSMISSÃO SILENCIOSA.
}

Hortência Lima Almeida $^{1}$; Maria Yana Guimarães Silva Freitas ${ }^{2}$; Brenda Fadigas Carvalho e Iago Barbosa Ribeiro $^{4}$

1. Voluntária PEVIC, Graduando em Enfermagem, Universidade Estadual de Feira de Santana, e-mail: hort.enfer@outlook.com

2. Orientadora, Departamento de Saúde, Universidade Estadual de Feira de Santana, e-mail: yana.guimaraess@gmail.com

3. Graduada em Enfermagem, Departamento de Saúde, Universidade Estadual de Feira de Santana, e-mail: brendafadigas@gmail.com

4. Graduando em Enfermagem, Departamento de Saúde, Universidade Estadual de Feira de Santana, e-mail: iagobarbosa04@gmail.com

PALAVRAS-CHAVE: Hanseníase, Prevenção, Transmissão.

\section{INTRODUÇÃO E JUSTIFICATIVA}

A hanseníase é uma doença infecciosa crônica causada pelo $M$. leprae, de dimensão social inigualável, que apresenta alta infectividade e baixa patogenicidade, o que significa que muitos se infectam, mas poucos adoecem (PARDINI; FREITAS, 2008). A transmissão do bacilo ocorre pelas vias aéreas superiores de pessoas multibacilares através do contato frequente e prolongado com as pessoas não tratadas. Em situações mais graves quando o agente infectante não encontra resistência para se multiplicar, este é disseminado para outros tecidos (ARAÚJO, 2003).

O diagnóstico precoce e o tratamento imediato contribuem para reduzir a incidência da doença, risco de incapacidades, sequelas e de deformidades. Mas em muitas situações a pessoa mesmo que complete o tratamento com êxito, posteriormente, desenvolve novos sinais e sintomas da doença, consideradas recidivas (AYRES; DUARTE; SIMONETTI, 2009).

A vigilância epidemiológica dos comunicantes visa detectar casos novos entre os contatos domiciliares do doente e programar medidas preventivas, a fim de contribuir para o rompimento da cadeia de transmissão da doença. Comparado à população em geral, o maior risco de adoecimento está entre os contatos domiciliares, o que indica que tanto o tipo de hanseníase quanto as distâncias do caso índice são fatores importantes para o risco da doença (LOBATO et al, 2016).

No Brasil as taxas de prevalência da hanseníase entre os contatos intradomiciliares da hanseníase são pelo menos 12 vezes maiores. E indicam a importância da vigilância dos contatos em um contexto de alta endemicidade, assim como expressaram que, embora a vigilância de contatos possa ser uma atividade difícil e árdua, ela pode trazer resultados palpáveis, tanto para o controle como para a eliminação da hanseníase (PINTO NETO, 2002).

Esse estudo focalizou a vigilância dos comunicantes intrafamiliares, meio onde pode ocorrer a transmissão da doença, afinal todo portador da doença primeiro foi um comunicante, então se faz necessário ter um enfoque de vigilância muito maior, por ser um grupo vulnerável, ajudando a detectar precocemente casos novos.

Assim, questiona-se: Qual a frequência de comunicantes examinados para prevenção da hanseníase em Feira de Santana no ano de 2016?

\section{OBJETIVO GERAL E ESPECÍFICOS}

GERAL:Avaliar o controle da hanseníase através da prevenção dos comunicantes. ESPECÍFICOS:Identificar o quantitativo de comunicantes examinados e não examinados no Programa de controle da hanseníase em Feira de Santana no ano de 2016;Analisar a contribuição do controle dos comunicantes para a prevenção da hanseníase.

\section{MATERIAIS E MÉTODOS}

Para verificar a ocorrência da avaliação dos comunicantes de pessoas com hanseníase foi realizado um estudo quantitativo transversal do tipo exploratório e descritivo, com os comunicantes de hanseníase no ano de 2016. 
O cenário de estudo da pesquisa foi o município de Feira de Santana que têm 627.477 mil habitantes (IGBE, 2017), que foram notificados em 201562 casos de hanseníase sendo 22 casos com hanseníase grau de incapacidade zero, $31 \mathrm{com}$ grau de incapacidade I, 04(quatro) com grau de incapacidade II, 03(três) não avaliados e 02(dois) ignorados o que indica retardo no diagnóstico. Dentre os 20 municípios da Bahia com maior incidência de hanseníase, Feira de Santana é $5^{\circ} \mathrm{com}$ a maior concentração de casos, portanto é considerado um município prioritário para o tratamento de hanseníase (SUVISA, 2015).

A população estudada foram os comunicantes das pessoas com hanseníase no ano de 2016 notificadas do tratamento no Programa de Controle de Hanseníase de Feira de Santana.

$\mathrm{O}$ atendimento as pessoas com hanseníase era realizado Centro de Saúde Especializado (CSE) Dr. Leone Coelho Lêda e a partir do primeiro semestre de 2017 com o processo de descentralização do serviço, o atendimento para casos mais simples foi transferido para as Unidades Básicas de Saúde (UBS) do município.

A coleta de dados foi realizada através do registro no Sistema de Informação de Agravos de Notificação (SINAN). Os dados do SINAN de 2016 sobre o programa da hanseníase foram disponibilizados pela Secretaria Municipal de Saúde através de um ofício, e assim que foram recebidos, analisamos o banco em comparação com a ficha de notificação de hanseníase e posteriormente, os dados foram tratados no programa STATA versão 14.0.

As variáveis foram categorizadas em 1 e 0 , onde 1 corresponde a risco e 0 como não risco, a categorização do risco foi feita a luz da literatura, as variáveis estudadas foram: contatos registrados, contatos examinados, forma clínica, classificação operacional e grau de incapacidade.

A variável contatos registrados, foram notificados de 0 a 10 contatos por pessoa, calculou-se a mediana, obtendo resultado 3,6 contatos por caso de hanseníase, foi utilizando cinco (5) como ponto de corte para categorização, ficando $\leq 5$ como risco (1).

A variável contatos examinados, foram notificados de 0 a 10 contatos por pessoa, calculou-se a mediana, obtendo resultado 3,6 contatos por caso de hanseníase, foi utilizando cinco (5) como ponto de corte para categorização, ficando $<=5$ como risco (1).

Foi calculado o Indicador operacional de Proporção de Examinados entre os contatos intradomiciliares de casos novos diagnosticados no ano, o denominador Contatos intradomiciliares de casos novos diagnosticados no ano que foram examinados, dividido por total de contatos intradomiciliares de casos novos diagnosticados no ano, multiplicado por 100. Como parâmetro de avaliação Bom: $\geq 75 \%$, Regular: 50 - 75\%, Precário: < 50\%.

O estudo foi pautado de acordo com os princípios éticos da Resolução nº 466 de dezembro de 2012 considerando o respeito à dignidade humana e pela especial proteção devida aos participantes das pesquisas científicas envolvendo seres humanos. Entretanto, por não envolver diretamente a participação de seres humanos não será necessária a utilização do Termo de Consentimento Livre e Esclarecido (TCLE), pois a pesquisa não envolverá diretamente a participação de seres humanos, entretendo será mantido o sigilo das informações.

Ainda que a pesquisa não demande o contato com os pacientes cujos prontuários foram selecionados para análise, pensa-se que o risco inerente ao estudo está relacionado à possibilidade de não manter o sigilo das informações coletadas. Entretanto em respeito à resolução 466/12 será mantido o anonimato dessas pessoas (BRASIL, 2012).

\section{RESULTADOS E DISCUSSÃO}

No período de 2016 foram registrados 246 comunicantes, dentre os quais 154 compareceram ao serviço e foram examinados, obtendo uma Proporção de Examinados entre os contatos intradomiciliares de casos 
novos diagnosticados no ano de 62, 6\%, correspondendo a uma avaliação regular das atividades de vigilância dos contatos.

Os contatos registrados foram 246 e variaram de 0 a 10 contatos, sendo $06(8 \%)$ dos casos índices não registraram nenhum contato, 20 (26,6\%) casos índices registraram 01 contato, 10(13,3\%) dos casos índices registram 02 contatos, $60(79,8 \%)$ casos índices registraram de 0 a 05 contatos.

Dos 246 contatos registrados, apenas 154 foram examinados, correspondendo a 62,6\%. $25(33,3 \%)$ casos índices não tiveram nenhum contato avaliado, 21 (28\%) casos índices tiveram 01 contato avaliado, $06(8 \%)$ casos índices tiveram 2 contatos avaliados , $66(87,9 \%)$ dos casos índices tiveram nenhum ou até 05 contatos avaliados.

Os dados encontrados no estudo mostram resultados melhores do que outros estudos que foram realizados. Mendonça (2017) em seu estudo sobre contatos no estado de Maranhão mostrou que de todos os contatos registrados, apenas 31,4\% foram examinados. Romanholo et al (2018) encontrou em seu estudo em Rondônia que apenas $41,6 \%$ dos contatos registrados foram examinados.

$\mathrm{Na}$ análise bivariada dos contatos registrados e examinados, os casos índices que registraram acima de 06 (seis) contatos, 9(nove) casos índices tiveram acima de 6(seis) contatos examinados. Os casos índices que registraram abaixo de 05(cinco) contatos, 66 casos índices tiveram de 0 a 5 contatos examinados.

A análise bivariada dos contatos examinados com a classificação operacional dos casos índices demonstrou que 54 (72\%) casos índices eram de classificação multibacilar.

Oliveira (2014) Em seu estudo sobre a ocorrência de hanseníase em contatos avaliados no estado do Maranhão encontrou que de 112 contatos que foram avaliados 07 desenvolveram a hanseníase. Trindade (2015) mostrou em seu estudo que de 190 contatos avaliados, 08 estavam tratando ou já fizeram o tratamento para a hanseníase, após o diagnóstico do caso índice.

Pacientes multibacilares são considerados a principal fonte de infecção e são, também, os mais susceptíveis a apresentar estados reacionais, sendo a forma paucibacilar (na qual poucos bacilos estão presentes) e a multibacilar na qual uma grande carga bacilar está presente nas lesões (LIMA el al, 2010).

\section{CONCLUSÃO}

Os dados encontrados nesse estudo demonstraram que as ações de vigilância dos contatos no município de Feira de Santana é regular, mas que ainda necessita de melhoras para que o alcance dos examinados seja maior, visto que boa parte dos casos índices são multibacilares, a forma mais grave e disseminante da doença.

O conhecimento fornecido por esse estudo da análise entre contatos domiciliares no município é de suma relevância, pois pode subsidiar para a elaboração de medidas e estratégias para modificar o quadro da doença no município.

A avaliação dos contatos possibilita que ao aparecimento de sinais e sintomas sugestivos de hanseníase identifiquem precocemente casos novos da doença, e possibilitem que a cadeia de transmissão da doença seja quebrada, além da prevenção de possíveis incapacidades.

Considerado a importância epidemiológica dos contatos e as limitações do estudo, recomenda-se intensificar ações de acompanhamento e busca ativa dos mesmos, atividade de educação em saúde sobre aspectos etiológicos da doença para a população em geral e em especial para as famílias com casos diagnosticados, além de novos estudos sobre esse grupo visto que a sua importância epidemiologia na manutenção e controle da doença. 


\section{REFERÊNCIAS}

OMS. Estratégia mundial de eliminação da lepra 2016-2020: Acelerar a ação para um mundo sem lepra. ISBN 97892-9022-520-1, 2016.

ARAÚJO AERA, AQUINO DMC, GOULART IMB, PEREIRA SRF, FIGUEIREDO IA, SERRA HO. Complicações neurais e incapacidades em hanseníase em capital do nordeste brasileiro com alta endemicidade. Rev Bras Epidemiol. 2014

AYRES, J, A; DUARTE, M, T; SIMONETTI, J, P. Consulta de enfermagem: estratégia de cuidado ao portador de hanseníase em atenção primária. Texto Contexto Enfermagem, Florianópolis, v.18, n.1, p. 100-107, jan-mai, 2009. BRASIL. Boletim Epidemiológico. Secretaria de Vigilância em Saúde. Ministério da Saúde - Brasil, 2018.

BRASIL. Resolução 466 de 12 de 12 de Dezembro de 2012. Brasilia:

http://conselho.saude.gov.br/resolucoes/2012/Reso2466.pdf.Acessado em 27 de março de 2016.

Ministério da Saúde. Secretaria de Vigilância em Saúde (SVS). Hanseníase; 2014. Disponível em <

http://portalsaude.saude.gov.br/index.php/o-ministerio/principal/secretarias/svs/hanseniase>. Acesso 23/03/2015.

COELHO,N.M.B.Caracterização dos Casos de Recidiva de Hanseníase diagnosticados entre 1994 e 2010 no município de Rondonópolis-MT. 2013. 48f. Dissertação de Mestrado. Universidade Católica de Góias. Góias. 2013.

DINIZ, L.M et al. Estudo retrospectivo de recidiva da hanseníase no Estado do Espírito Santo. Revista da Sociedade

Brasileira de Medicina Tropical. v. 4, n.4, p.420-424, jul-ago, 2009.

HERCULES,F.M. Recidiva de Hanseníase no município de Duque de Caxias. 2004. 158f. Dissertação de Mestrado.

Universidade Federal do Rio de Janeiro. Rio de Janeiro. 2004

KLEINBAUM DG, SULLIVAN K, BARKER N. A pocket guide to epidemiology. Springer, 2006. ISBN 0387459642.

MONTEIRO LD, ALENCAR CHM, BARBOSA JC, BRAGA KP, CASTRO MD, HEUKELBACH J. Incapacidades físicas em pessoas acometidas pela hanseníase no período pós-alta da poliquimioterapia em um município no norte do Brasil. Cad Saúde Pública. 2013.

PARDINI, A.G; FREITAS, A. A Cirurgia da Mão: Lesões Não- Traumáticas. 2 ed. Rio de Janeiro: Medbook, 2008. PAGANO M, GAUVREAU K.Princípios de Bioestatística. São Paulo: Editora Pioneira Thomson Learning;2004. 